\title{
Carcinosarcoma of Breast with Hormone Receptor and HER2/neu Positivity
}

\section{ABSTRACT}

Carcinosarcoma is an extremely rare tumor of the breast comprising of two distinct cell lines i.e. the epithelial and mesenchymal. It is a highly aggressive tumor with a poor prognosis compared to other breast cancers with a reported incidence of less than 1\% of all the breast cancers. A 35 year old female presented to surgical outpatient with a fungating mass in the right breast where cytological analysis from the affected areas revealed spindle cells of malignant potential.
A modified radical mastectomy was performed and following histopathological examination of the excised tissue, a final diagnosis of carcinosarcoma of breast was concluded. Immunological staining done showed a negative staining for PR and positive staining for ER, HER-2/neu and CK receptors, as against the conventional triple negative staining pattern. Along with a rare staining pattern of this rare tumor reported in literature, this case merits presentation owing to its diagnostic and therapeutic challenges.

\section{INTRODUCTION}

The World Health Organization (WHO) has defined carcinosarcoma as a tumor composed of intimately admixed malignant epithelial and stromal components [1]. These are categorized under primary sarcomas of the breast and constitute nearly 0.6 to $1.2 \%$ of the total number of malignant tumors of the breast [2]. It is suggested by the evidences that these tumors are similar to monoclonal tumors that develop from malignant transformation or myofibroblastic metaplasia of epithelial cells. Thus, the term biphasic sarcomatoid carcinoma is used to describe all breast carcinomas with overt carcinomatous and sarcomatous features. When only epithelial component is detected at electron microscopy or immunohistochemistry it is considered as Monophasic sarcomatoid carcinoma' [3]. These tumors do not express the estrogen or progesterone receptors and HER-2/neu oncogene. Due to this "triple negative" phenotype, such tumors tend to be more aggressive and fail to respond to hormone therapy and herceptin [4]. Immunohistochemical features of carcinosarcoma breast shows immunoreactivity for keratin (55\%), vimentin (98\%), actin (77\%) and S-100 protein (55\%) [5]. The case merits presentation because of its rarity and difficulty to diagnose mainly due to partial receptor positivity.

\section{CASE STUDY}

A 36 year old female presented to the Surgical OPD of with a progressively increasing fungating mass in right breast since past four months. Patient had off and on sero-purulent discharge with local rise in temperature and tenderness since past two months. There was no past history of surgery / radiotherapy / chemotherapy. Physical examination revealed a firm mass of $7 \times 5.5 \mathrm{~cm}$ beneath the nipple. Overlying skin was ulcerated with retraction of nipple. No metastasis to axillary or cervical lymph nodes was obvious on clinical examination. A fine needle aspiration biopsy was performed which revealed malignant cells of mesenchymal cell line. A modified radical mastectomy was performed and axillary lymph nodes up to level two were sent for histopathological evaluation.

Gross examination of the specimen showed a large, well defined growth, measuring $7 \times 5.5 \times 5 \mathrm{~cm}$. The skin surface showed gray white ulcer with retraction of nipple. The cutsection of lesion was grey white with a whorled appearance [Table/Fig-1].

Microscopic Examination of the tumor showed intermingled carcinomatous and sarcomatous areas. At the periphery of the sarcomatous area were present nests of atypical ductal epithelial cells [Table/Fig -2]. The sarcomatous areas showed sheets of spindle shaped exhibiting pleomorphism with 

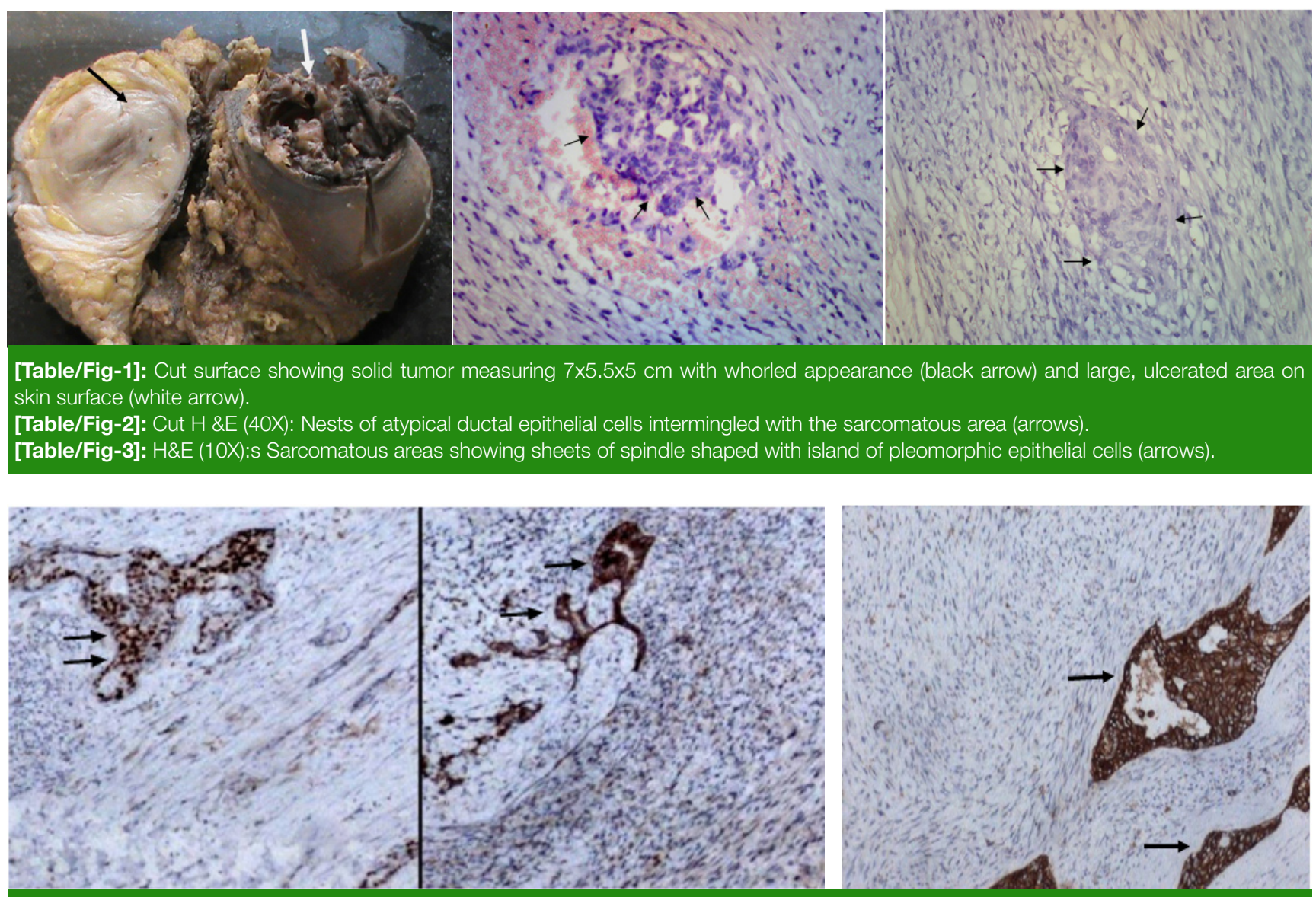

[Table/Fig-4]: Nest of epithelial cells exhibiting ER and HER2/neu receptor positivity (arrows).

[Table/Fig-5]: Island of epithelial cells exhibiting cytokeratin positivity (arrows).

hyperchromatic nuclei but there was no specific sarcomatous feature [Table/Fig-3]. Mitotic figures were frequent in both the carcinomatous and sarcomatous areas. Large areas of necrosis were also seen. Histopathological examination of the 12 lymph nodes dissected from the axillary tail was consistent with 'reactive hyperplasia'.

Immunohistochemistry: Tumor cells revealed positive staining for estrogen, HER2/neu [Table/Fig-4] and cytokeratin onco-receptor [Table/Fig-5] while negative staining was seen for progesterone receptor. Post-surgery, the patient received chemotherapy with cyclophosphamide, adriamycin, 5 -flurouracil, and was in satisfactory health condition at 8 months follow-up.

\section{DISCUSSION}

Metaplastic breast carcinomas (MBC) are a heterogeneous group of malignant lesions comprising $<1 \%$ of all invasive breast cancers [6]. The origin of breast carcinosarcoma is considered to be controversial. Recently, the use of gene profiling has identified most receptor-negative breast cancers as a unique 'basal' form of breast cancer thought to arise from progenitor cells in the ductal epithelium [4]. Metaplastic carcinomas have some 'sarcoma-like' features like node

negativity and the inverse relation of tumor size with the outcome.

So, possibly the metaplastic carcinomas is a type of breast adenocarcinoma originating from a basal progenitor cell that comprises of both morphologic and clinical sarcomatoid characteristics. MBC have also been reported to develop in existing lesions of cystosarcoma phyllodes, fibroadenoma or cystic nature [4]. Pathologic classification of metaplastic carcinomas remains problematic but the disease is broadly divided into two main groups: (1) homogeneous spindle cell/sarcomatoid carcinoma, and (2) heterogeneous carcinosarcoma/ carcinoma with sarcomatous differentiation (osseous, chondroid and rhabdoid). Pure epithelial malignant tumors with metaplasia, such as adenosquamous and pure squamous cell carcinoma are also considered as MBC subtypes [6].

Pathologic classification of MBC is challenging due to the diverse histological presentation and rarity of the diagnosis. 
MBC with a prominent spindle cell component contains atypical spindled cells with a fascicular or storiform arrangement. The degree of cytologic atypia however, can vary from markedly pleomorphic tumors resembling high grade sarcomas to mild cellular atypia resembling benign fibrous lesions. The presence and amount of the associated ductal carcinomatous component can also vary. Immunohistochemical evaluation is essential to demonstrate epithelial differentiation in the diagnosis of $\mathrm{MBC}$ with a homogenous spindle cell population.

The differential diagnosis of $\mathrm{MBC}$ includes infiltrating ductal carcinoma with a pseudo-sarcomatous stroma, though the sarcomatous area is vimentin negative. Sarcomatoid carcinoma /spindle cell carcinoma also resembles carcinosarcoma with both the epithelial and sarcoma like components, though here a gradual transition or separation can be distinguished while in carcinosarcoma, both these components are intimately admixed. Also, in sarcomatoid carcinomas, the sarcomatous areas exhibit CK positivity [7].

A differential diagnosis of cystosarcoma phylloides with malignant transformation of epithelial component was a close possibility, but was ruled out as the tumor lacked the curved elongated ducts with stromal leaf like projections. Matrix producing carcinoma was the next differential diagnostic possibility, but as the tumor cells lacked chondroid and/or osseous stromal matrix, the same was excluded. Other less common differential diagnosis included stromal sarcoma, pure sarcomas like fibrosarcoma and malignant fibrous histiocytoma, which were excluded on the basis of their histological characteristics $[7,8]$.

It is seen that metaplastic carcinomas are ER, PR and HER-2/ neu negative, but have also been found to infrequently express hormone receptors with estrogen and or progesterone (ER/ $\mathrm{PR}$ ) positivity ranging from 0 to $17 \%$ in many series [6]. We observed that tumor cells exhibited positive staining for ER, HER-2/neu and cytokeratin in our case.

The prognosis of carcinosarcoma breast is worse than classical breast carcinomas and these carry high risk of recurrence following primary surgery owing to its aggressive nature. A review of 16 publications on carcinosarcoma breast by Beatty et al., has revealed a five-year overall survival ranging from 49-68\% [9]. The five year survival rates for TNM clinical Stages I, II, and III was described to be 100\%, 63\%, and 35\% respectively by Wargotz et al., [10].

Recently, Hennessy et al., collected information from various databases and compared clinical features and survival rates in biphasic metaplastic sarcomatoid carcinoma (MSC) and carcinosarcoma in 100 and 98 cases respectively [11]. Triple negative breast cancer responds only to chemotherapy (e.g. taxane). Recently, epidermal growth factor receptor (EGFR) inhibitors such as gefitinib (ZD1839, Iressa) and cetuximab (Erbitux) have been introduced for the management of carcinosarcoma and are presently on trial basis [12]. Hence forth, obtaining an accurate diagnosis of carcinosarcoma breast is essential to optimally tailor adjuvant therapy towards this aggressive tumor.

\section{CONCLUSION}

Carcinosarcoma is a diagnosis of exclusion that exhibits triple negativity on immunochemistry. It is a treatment refractory tumor with aggressive course and poor outcomes mimicking undifferentiated receptor-negative adenocarcinoma of the breast. Current literature shows that controversies still exist regarding their histogenesis and classification. Tripple negativity on hormone receptor analysis is the strongest indicator of carcinosarcoma, although possibility of ER and HER2/neu positivity may be observed in rare cases. Immunochemistry should be performed in all cases to aid in diagnosis, assign the correct prognosis and guide appropriate treatment to the patient.

\section{REFERENCES}

[1] Srinivas V, Harjai MM, Subramanya $H$, Rajaram T, Rai R. Carcinosarcoma of the Breast With An Unusual Secretory Carcinoma as the Carcinomatous Component. Medical Journal Armed forces India. 2004; 60(4): 410-12.

[2] Gutman H, Pollock RE, Janjan NA, Johnston DA. Biologic distinctions and therapeutic implications of sarcomatoid metaplasia of epithelial carcinoma of the breast. J Am Coll Surg 1995; 180(2):193-99.

[3] Ilhan E,Vardar E, Ozkok G, Sezgin A, Sahin S, Tekera K. A Rare Tumour of the Breast: Carcinosarcoma. J Clin Med Res 2010;2(2):96-98.

[4] Atahan K, Gür S, Tarcan E, Çökmez A, Yi it S. Carcinosarcoma of the breast. The Journal of Breast Health. 2007; 3(2): 85-86.

[5] Abbasi MA, Mahmood H, Faheem M, Khan AK, Irfan J. Carcinosarcoma of the Breast. Journal of the College of Physicians and Surgeons Pakistan. 2012;22 (5): 333-34.

[6] Patnayak R, Rajasekhar S, Narendra H, Narayan ML, Kalawat TC, Silpa K, Jena A. Carcinoma or sarcoma of the breast. $J$ Basic Clin Reprod Sci 2014;3:121-25.

[7] Barnes PJ, Boutilier R, Chiasson D, Rayson D. Metaplastic breast carcinoma: clinical- pathologic characteristics and HER2/neu expression. Breast Cancer Research and Treatment. 2005;91(2):173-78.

[8] Kurian KM, Al-Nafussi A: Sarcomatoid/metaplastic carcinoma of the breast: a clinic-pathological study of 12 cases. Histopathology 2002; 40(1):58-64.

[9] Beatty JD, Atwood M, Tickman R, Reiner M. Metaplastic breast cancer: clinical significance. Am J Surg. 2006; 191(5):657-64.

[10] Wargotz ES, Norris HJ. Metaplastic carcinomas of the breast. III. Carcinosarcoma. Cancer 1989 Oct 1; 64(7):1490-99.

[11] Hennessy BT, Giordan S,Broglio K, Duan Z, Trent J, Buchholz TA et al. Biphasic metaplastic sarcomatoid carcinoma of the breast. Annals of Oncology. 2006;17(4): 605-13. doi:10.1093/annonc/ mall006.

[12] Esses KM, Hagmaier RM, Blanchard SA, Lazarchick JJ, Iriker A. Carcinosarcoma of the breast: two case reports and review of the literature. Case Journal. 2009; 2(1):15. doi:10.1186/17571626-2-15. 


\section{AUTHOR(S):}

1. Dr. Deepti Agarwal

2. Dr. Vijay Malik

3. Dr. Ruchi Agarwal

4. Dr. M.K. Garg

5. Dr. Shilpa Garg

\section{PARTICULARS OF CONTRIBUTORS:}

1. Associate Professor, Department of Pathology, BPS Govt. Medical College for Women, Khanpur Kalan, Sonipat, Haryana, India.

2. Assistant Professor, Department of Surgery, BPS Govt. Medical College for Women, Khanpur Kalan, Sonipat, Haryana, India.

3. Assistant Professor, Department of Pathology, BPS Govt. Medical College for Women, Khanpur Kalan, Sonipat, Haryana, India.
4. Professor and Head of Department, Department of Surgery, BPS Govt. Medical College for Women, Khanpur Kalan, Sonipat, Haryana, India.

5. Assistant Professor, Department of Pathology, Pt. BD Sharma PGIMS Rohtak, Haryana, India.

\section{NAME, ADDRESS, E-MAIL ID OF THE} CORRESPONDING AUTHOR:

Dr. Deepti Agarwal,

Associate Professor, Department of Pathology, BPS Govt. Medical College for Women, Khanpur Kalan, Sonipat,

Haryana, India.

E-mail: deeptiagarwal@rocketmail.com

FINANCIAL OR OTHER COMPETING INTERESTS: None.

Date of Publishing: Jan 05, 2016 